\title{
On the Numerical Solution of Differential-Algebraic Equations with Hessenberg Index-3
}

\author{
Melike Karta ${ }^{1}$ and Ercan Çelik ${ }^{2}$ \\ ${ }^{1}$ Department of Mathematics, Faculty of Art and Science, A ğrn Ibrahim Ceçen University, \\ 04100 Agri, Turkey \\ ${ }^{2}$ Department of Mathematics, Atatürk University Faculty of Science, 25240 Erzurum, Turkey
}

Correspondence should be addressed to Ercan Çelik, ercelik@atauni.edu.tr

Received 12 April 2011; Revised 18 October 2011; Accepted 9 November 2011

Academic Editor: Antonia Vecchio

Copyright (C) 2012 M. Karta and E. Çelik. This is an open access article distributed under the Creative Commons Attribution License, which permits unrestricted use, distribution, and reproduction in any medium, provided the original work is properly cited.

Numerical solution of differential-algebraic equations with Hessenberg index-3 is considered by variational iteration method. We applied this method to two examples, and solutions have been compared with those obtained by exact solutions.

\section{Introduction}

Many important mathematical models can be expressed in terms of differential-algebraic equations (DAEs). Many physical problems are most easily initially modeled as a system of differential-algebraic equations (DAEs) [1]. Some numerical methods have been developed, using both BDF [1-3] and implicit Runge-Kutta methods [1], Padé and Chebysev approximations method [4-6]. These methods are only directly suitable for low-index problems and often require that the problem, have special structure. Although many important applications can be solved by these methods, there is a need for more general approaches. There are many new publication in the field of analytical sueveys such as [7-10]. The variational iteration method (VIM) was developed by He in [11]. The method is used by many researchers in a variety of scientific fields. The method has been proved by many authors [12-16] to be reliable and efficient for a variety of scientific applications, linear and nonlinear as well.

The most general form of a DAE is given by

$$
F\left(t, x, x^{\prime}\right)=0,
$$


where $\partial F / \partial x^{\prime}$ may be singular. The rank and structure of this Jacobian matrix may depend, in general, on the solution $x(t)$, and for simplicity we will always assume that it is independent of $t$. The important special case is of a semiexplicit DAE or an ODE with constraints:

$$
\begin{aligned}
& x^{\prime}=f(t, x, z), \\
& 0=g(t, x, z) .
\end{aligned}
$$

This is a special case of (1.1). The index is 1 if $\partial g / \partial z$ is nonsingular, because then one differentiation of $(1.2 b)$ yields $z^{\prime}$ in principle. For the semi-explicit index-1 DAE we can distinguish between differential variables $x(t)$ and algebraic variables $z(t)$ [1]. The algebraic variables may be less smooth than the differential variables by one derivative. In the general case, each component of $x$ may contain a mix of differential and algebraic components, which makes the numerical solution of such high-index problems much harder and riskier.

\section{Special Differential-Algebraic Equations (DAEs) Forms}

Most of the higher-index problems encountered in practice can be expressed as a combination of more restrictive structures of ODEs coupled with constraints. In such systems the algebraic and differential variables are explicitly identified for higher-index DAEs as well, and the algebraic variables may all be eliminated using the same number of differentiations. These are called Hessenberg forms of the DAE and are given below. In this paper, the variational iteration method has been proposed for solving differential-algebraic equations with Hessenberg index-3.

\subsection{Hessenberg Index-1}

One has

$$
\begin{aligned}
& x^{\prime}=f(t, x, z), \\
& 0=g(t, x, z) .
\end{aligned}
$$

Here the Jacobian matrix function $g_{z}$ is assumed to be nonsingular for all $t$. This is also often referred to as a semi-explicit index-1 system. Semi-explicit index-1 DAEs are very closely related to implicit ODEs.

\subsection{Hessenberg Index-2}

One has

$$
\begin{gathered}
x^{\prime}=f(t, x, z), \\
0=g(t, x) .
\end{gathered}
$$

Here the product of Jacobians $g_{x} f_{z}$ is nonsingular for all $t$. Note the absence of the algebraic variables $z$ from the constraints (2.2b). This is a pure index-2 DAE, and all algebraic variables play the role of index-2 variables. 


\subsection{Hessenberg Index-3}

One has

$$
\begin{gathered}
x^{\prime}=f(t, x, y, z), \\
y^{\prime}=g(t, x, y), \\
0=h(t, y) .
\end{gathered}
$$

Here the product of three matrix functions $h_{y} g_{x} f_{z}$ is nonsingular.

The index of a Hessenberg DAE is found, as in the general case, by differentiation. However, here only algebraic constraints must be differentiated.

\section{He's Variational Iteration Method (VIM)}

Consider the differential equation

$$
L u+N u=g(x)
$$

where $L$ and $N$ are linear and nonlinear operators, respectively, and $g(x)$ is the source inhomogeneous term. In [11], He proposed the variational iteration method where a correction functional for (3.1) can be written as

$$
u_{n+1}(x)=u_{n}(x)+\int_{0}^{x} \lambda(t)\left(L u_{n}(t)+N \tilde{u}_{n}(t)-g(t)\right) d t
$$

where $\lambda$ is a general Lagrange's multiplier, which can be identified optimally via the variational theory and $\tilde{u}_{n}$ as a restricted variation which means $\delta \tilde{u}_{n}=0$. It is to be noted that the Lagrange multiplier $\lambda$ can be a constant or a function.

The variational iteration method should be employed by following two essential steps. It is required first to determine the Lagrange multiplier $\mathcal{\lambda}$ that can be identified optimally via integration by parts and by using a restricted variation. Having $\lambda$ determined, an iteration formula, without restricted variation, should be used for the determination of the successive approximations $u_{n+1}(x), n \geq 0$, of the solution $u(x)$. The zeroth approximation $u_{0}$ can be any selective function. However, using the initial values $u(0), u^{\prime}(0)$, and $u^{\prime \prime}(0)$ are preferably used for the selective zeroth approximation $u_{0}$ as will be seen later. Consequently, the solution is given by

$$
u_{n}(x)=\lim _{n \rightarrow \infty} u_{n}(x)
$$

\subsection{First-Order ODEs}

We first start our analysis by studying the first-order linear ODE of a standard form

$$
u^{\prime}+p(x) u=q(x), \quad u(0)=\alpha .
$$


The VIM admits the use of the correction functional for this equation by

$$
u_{n+1}(x)=u_{n}(x)+\int_{0}^{x} \lambda(t)\left(u_{n}^{\prime}(t)+p(t) \tilde{u}_{n}(t)-g(t)\right) d t
$$

where $\lambda$ is the lagrange multiplier, that in this method may be a constant or a function, and $\tilde{u}_{n}$ is a restricted value where $\delta \tilde{u}_{n}=0$. we have

Taking the variation of both sides of (3.5) with respect to the independent variable $u_{n}$

$$
\delta u_{n+1}(x)=\delta u_{n}(x)+\delta\left(\int_{0}^{x} \lambda(t)\left(u_{n}^{\prime}(t)+p(t) \tilde{u}_{n}(t)-q(t) d t\right)\right)
$$

that gives

$$
\delta u_{n+1}(x)=\delta u_{n}(t)+\delta\left(\int_{0}^{x} \lambda(t) u_{n}^{\prime}(t) d t\right)
$$

obtained upon using $\delta \tilde{u}_{n}=0$ and $\delta q(t)=0$. Integrating the integral of (3.6) by parts we obtain

$$
\delta u_{n+1}=\delta u_{n}+\delta \lambda u_{n}(x)-\delta \int_{0}^{x} \lambda^{\prime} u_{n} d t
$$

or equivalently

$$
\delta u_{n+1}=\delta\left(1+\left.\lambda\right|_{t=x}\right) u_{n}-\delta \int_{0}^{x} \lambda^{\prime} u_{n} d t
$$

The extremum condition of $u_{n+1}$ requires that $u_{n+1}=0$. This means that the left hand side of (3.9) is 0 , and as a result the right hand side should be 0 as well. This yields the stationary conditions

$$
\begin{gathered}
1+\lambda_{t=x}=0, \\
\left.\lambda^{\prime}\right|_{t=x}=0 .
\end{gathered}
$$

This in turn gives

$$
\lambda=-1
$$

Substituting this value of the lagrange multiplier into the functional (3.5) gives the iteration formula

$$
u_{n+1}(x)=u_{n}(x)-\int_{0}^{x}\left(u_{n}^{\prime}(t)+p(t) u_{n}(t)-q(t)\right) d t
$$


obtained upon deleting the restriction on $u_{n}$ that was used for the determination of $\lambda$. Considering the given condition $u(0)=\alpha$, we can select the zeroth approximation $u_{o}=\alpha$. Using the selection into (3.12) we obtain the following successive approximations:

$$
\begin{gathered}
u_{0}(t)=\alpha, \\
u_{1}(x)=\alpha-\int_{0}^{x}\left(u_{0}^{\prime}(t)+p(t) u_{0}(t)-q(t)\right) d t \\
u_{2}(x)=u_{1}(x)-\int_{0}^{x}\left(u_{1}^{\prime}(t)+p(t) u_{1}(t)-q(t)\right) d t \\
u_{3}(x)=u_{2}(x)-\int_{0}^{x}\left(u_{2}^{\prime}(t)+p(t) u_{2}(t)-q(t)\right) d t \\
\vdots \\
u_{n+1}=u_{n}(x)-\int_{0}^{x}\left(u_{n}^{\prime}(t)+p(t) u_{n}(t)-q(t)\right) d t .
\end{gathered}
$$

Recall that

$$
u(x)=\lim _{n \rightarrow \infty} u_{n+1}(x)
$$

that may give the exact solution if a closed form solution exists, or we can use the $(n+1)$ th approximation for numerical purposes.

\section{Applications}

Example 4.1. We first considered the following differential-algebraic equations with Hessenberg index-3 form:

$$
\begin{gathered}
x_{2}^{\prime}+x_{1}-1=0, \\
x x_{2}^{\prime}+x_{3}^{\prime}+2 x_{2}-2 x=0, \\
x x_{2}+x_{3}-e^{x}=0
\end{gathered}
$$

with initial conditions

$$
\left(\begin{array}{l}
x_{1}(0) \\
x_{2}(0) \\
x_{3}(0)
\end{array}\right)=\left(\begin{array}{c}
0 \\
-1 \\
1
\end{array}\right) .
$$


The exact solutions are

$$
x_{1}(x)=e^{x}-1, \quad x_{2}(x)=2 x-e^{x}, \quad x_{3}(x)=(1+x) e^{x}-2 x^{2},
$$

where $x_{2}, x_{3}$ represent the differential variables and $x_{1}$ represents the algebraic variables. After three times of differentiation of (4.1) we have the following ODE system:

$$
\begin{gathered}
x_{1}^{\prime}=e^{x}, \\
x_{2}^{\prime}=2-e^{x}, \\
x_{3}^{\prime}=x x_{1}-2 x_{2}+x .
\end{gathered}
$$

Differential-algebraic equation (DAE) is a Hessenberg index-3 form.

To solve system (4.4), we can construct following correction functionals:

$$
\begin{gathered}
x_{1}^{(n+1)}(x)=x_{1}^{(n)}(x)+\int_{0}^{x} \lambda_{1}(t)\left(x_{1}^{\prime(n)}(t)-e^{t}\right) d t \\
x_{2}^{(n+1)}(x)=x_{2}^{(n)}(x)+\int_{0}^{x} \lambda_{2}(t)\left(x_{2}^{\prime(n)}(t)-2+e^{t}\right) d t \\
x_{3}^{(n+1)}(x)=x_{3}^{(n)}(x)+\int_{0}^{x} \lambda_{3}(t)\left(x_{3}^{\prime(n)}(t)-t \tilde{x}_{1}^{(n)}+2 \tilde{x}_{2}^{(n)}-t\right) d t
\end{gathered}
$$

where $\lambda_{1}(t), \lambda_{2}(t)$, and,$\lambda_{3}(t)$ are general Lagrange multipliers and $\tilde{x}_{1}^{(n)}, \tilde{x}_{2}^{(n)}$ denote restricted variations, that is, $\delta \tilde{x}_{1}^{(n)}=\delta \tilde{x}_{2}^{(n)}=0$.

Making the above correct functional stationary,

$$
\begin{gathered}
\delta x_{1}^{(n+1)}(x)=\delta x_{1}^{(n)}(x)+\delta \int_{0}^{x} \lambda_{1}(t)\left(x_{1}^{\prime(n)}(t)-e^{t}\right) d t \\
\delta x_{2}^{(n+1)}(x)=\delta x_{2}^{(n)}(x)+\delta \int_{0}^{x} \lambda_{2}(t)\left(x_{2}^{\prime(n)}(t)-2+e^{t}\right) d t \\
\delta x_{3}^{(n+1)}(x)=\delta x_{3}^{(n)}(x)+\delta \int_{0}^{x} \lambda_{3}(t)\left(x_{3}^{\prime(n)}(t)-t \tilde{x}_{1}^{(n)}(t)+2 \tilde{x}_{2}^{(n)}(t)-t\right) d t \\
\delta x_{1}^{(n+1)}(x)=\delta x_{1}^{(n)}(x)+\left.\delta \lambda_{1}(t) x_{1}^{(n)}(t)\right|_{0} ^{x}-\int_{0}^{x} \lambda_{1}^{\prime}(t) \delta x_{1}^{(n)}(t) d t=0 \\
\delta x_{2}^{(n+1)}(x)=\delta x_{2}^{(n)}(x)+\left.\delta \lambda_{2}(t) x_{2}^{(n)}(t)\right|_{0} ^{x}-\int_{0}^{x} \lambda_{2}^{\prime}(t) \delta x_{2}^{(n)}(t) d t=0 \\
\delta x_{3}^{(n+1)}(x)=\delta x_{3}^{(n)}(x)+\left.\delta \lambda_{3}(t) x_{3}^{(n)}(t)\right|_{0} ^{x}-\int_{0}^{x} \lambda_{3}^{\prime}(t) \delta x_{3}^{(n)}(t) d t=0 .
\end{gathered}
$$


Its stationary conditions can be obtained as follows:

$$
\begin{gathered}
\lambda_{1}^{\prime}(t)=\lambda_{2}^{\prime}(t)=\lambda_{3}^{\prime}(t)=0, \\
1+\left.\lambda_{1}(t)\right|_{t=x}=1+\left.\lambda_{2}(t)\right|_{t=x}=1+\left.\lambda_{3}(t)\right|_{t=x}=0 .
\end{gathered}
$$

The Lagrange multipliers can be identified as follows:

$$
\lambda_{1}(t)=\lambda_{2}(t)=\lambda_{3}(t)=-1
$$

and the following multipliers can be obtained as

$$
\begin{gathered}
x_{1}^{(n+1)}(x)=x_{1}^{(n)}(x)-\int_{0}^{x}\left(x_{1}^{\prime(n)}(t)-e^{t}\right) d t \\
x_{2}^{(n+1)}(x)=x_{2}^{(n)}(x)-\int_{0}^{x}\left(x_{2}^{\prime(n)}(t)-2+e^{t}\right) d t \\
x_{3}^{(n+1)}(x)=x_{3}^{(n)}(x)-\int_{0}^{x}\left(x_{3}^{\prime(n)}(t)-t \tilde{x}_{1}^{(n)}+2 \tilde{x}_{2}^{(n)}-t\right) d t .
\end{gathered}
$$

Beginning with $x_{1}^{(0)}(x)=0, x_{2}^{(0)}(x)=-1, x_{3}^{(0)}(x)=1$ by the iteration formula (4.9), we have

$$
\begin{gathered}
x_{1}^{*}(x)=x+\frac{1}{2} x^{2}+\frac{1}{6} x^{3}+\frac{1}{24} x^{4}+\frac{1}{120} x^{5}+\frac{1}{720} x^{6}+\frac{1}{5040} x^{7}+\frac{1}{40320} x^{8}-\frac{1}{362880} x^{9}+\cdots, \\
x_{2}^{*}(x)=-1+x-\frac{1}{2} x^{2}-\frac{1}{6} x^{3}-\frac{1}{24} x^{4}-\frac{1}{120} x^{5}-\frac{1}{720} x^{6}-\frac{1}{5040} x^{7}-\frac{1}{40320} x^{8}-\frac{1}{362880} x^{9}-\cdots, \\
x_{3}^{*}(x)=1+2 x-\frac{1}{2} x^{2}+\frac{2}{3} x^{3}+\frac{5}{24} x^{4}+\frac{1}{20} x^{5}+\frac{7}{720} x^{6}+\frac{1}{630} x^{7}+\frac{1}{4480} x^{8}+\frac{1}{36288} x^{9}+\cdots
\end{gathered}
$$

Example 4.2. One has

$$
\left(\begin{array}{lll}
1 & 0 & 0 \\
0 & 1 & 0 \\
0 & 0 & 0
\end{array}\right)\left(\begin{array}{l}
v_{1}^{\prime} \\
v_{2}^{\prime} \\
v_{3}^{\prime}
\end{array}\right)+\left(\begin{array}{ccc}
1 & 1 & x \\
e^{x} & x+1 & 0 \\
0 & x^{2} & 0
\end{array}\right)\left(\begin{array}{l}
v_{1} \\
v_{2} \\
v_{3}
\end{array}\right)=\left(\begin{array}{c}
2 x \\
x^{2}+x+2 \\
x^{3}
\end{array}\right)
$$

with initial conditions

$$
x(0)=\left(\begin{array}{l}
1 \\
0 \\
1
\end{array}\right)
$$


Table 1: Numerical solution of $x_{1}(x)$.

\begin{tabular}{lccc}
\hline$x$ & $x_{1}(x)$ & $x_{1}^{*}(x)$ & $\left|x_{1}(x)-x_{1}^{*}(x)\right|$ \\
\hline 0.0 & 0 & 0 & 0 \\
0.1 & 0.105170918 & 0.1051709181 & $0.1 \times 10^{-9}$ \\
0.2 & 0.221402758 & 0.2214027582 & $0.2 \times 10^{-9}$ \\
0.3 & 0.349858808 & 0.3498588076 & $0.4 \times 10^{-9}$ \\
0.4 & 0.491824698 & 0.4918246977 & $0.3 \times 10^{-9}$ \\
0.5 & 0.648721271 & 0.6487212705 & $0.5 \times 10^{-9}$ \\
0.6 & 0.822118800 & 0.8221187987 & $0.13 \times 10^{-8}$ \\
0.7 & 1.013752707 & 1.013752699 & $0.8 \times 10^{-8}$ \\
0.8 & 1.225540928 & 1.225540897 & $0.31 \times 10^{-7}$ \\
0.9 & 1.459603111 & 1.459603006 & $0.105 \times 10^{-6}$ \\
1.0 & 1.718281828 & 1.718281527 & $0.301 \times 10^{-6}$ \\
\hline
\end{tabular}

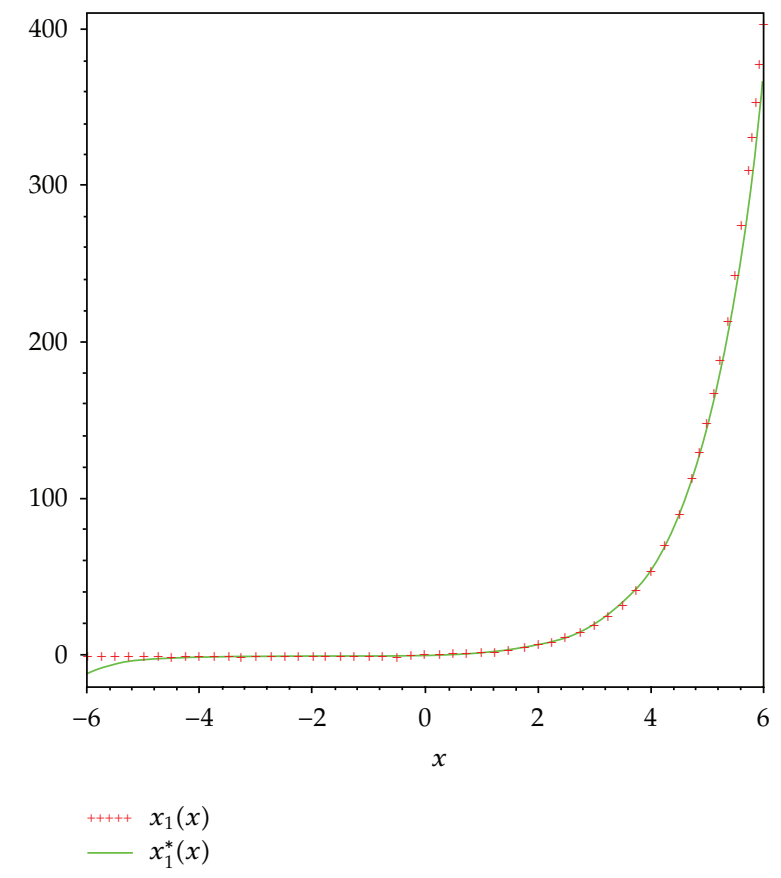

Figure 1: Values of $x_{1}(x)$ and its $x_{1}^{*}(x)$ variational iteration.

The exact solutions are $v_{1}(x)=e^{-x}, v_{2}(x)=x, v_{3}(x)=1$ :

$$
\begin{gathered}
v_{1}^{\prime}+v_{1}+v_{2}+x v_{3}-2 x=0 \\
v_{2}^{\prime}+e^{x} v_{1}+(x+1) v_{2}-x^{2}-x-2=0 \\
x^{2} v_{2}-x^{3}=0
\end{gathered}
$$


Table 2: Numerical solution of $x_{2}(x)$.

\begin{tabular}{lccc}
\hline$x$ & $x_{2}(x)$ & $x_{2}^{*}(x)$ & $\left|x_{2}(x)-x_{2}^{*}(x)\right|$ \\
\hline 0.0 & -1 & -1 & 0 \\
0.1 & -0.905170918 & -0.9051709181 & $0.1 \times 10^{-9}$ \\
0.2 & -0.821402758 & -0.8214027582 & $0.2 \times 10^{-9}$ \\
0.3 & -0.749858808 & -0.7498588076 & $0.4 \times 10^{-9}$ \\
0.4 & -0.691824698 & -0.6918246977 & $0.3 \times 10^{-9}$ \\
0.5 & -0.648721271 & -0.6487212705 & $0.5 \times 10^{-9}$ \\
0.6 & -0.622118800 & -0.6221187987 & $0.13 \times 10^{-8}$ \\
0.7 & -0.613752707 & -0.6137526992 & $0.78 \times 10^{-8}$ \\
0.8 & -0.625540928 & -0.6255408967 & $0.313 \times 10^{-7}$ \\
0.9 & -0.659603111 & -0.6596030066 & $0.1044 \times 10^{-6}$ \\
1.0 & -0.718281828 & -0.7182815256 & $0.3024 \times 10^{-6}$ \\
\hline
\end{tabular}

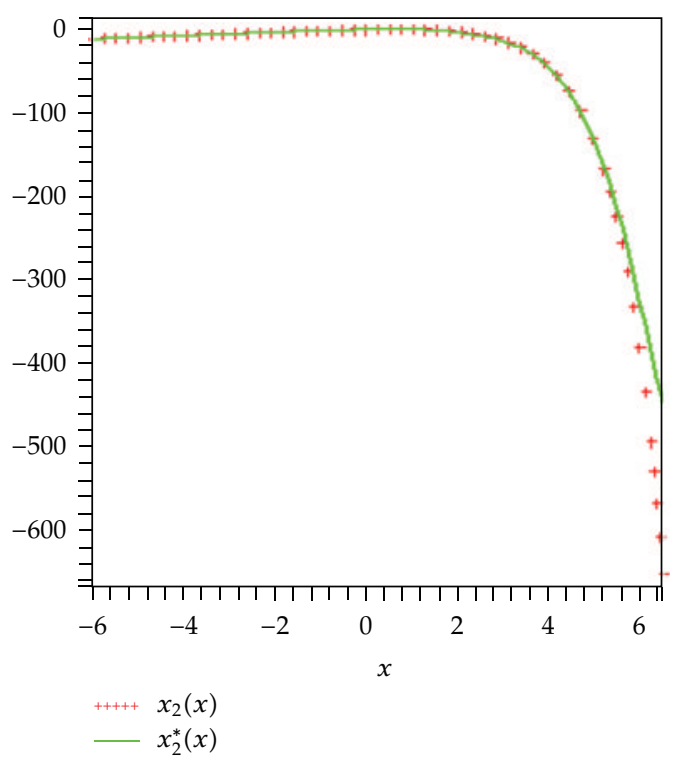

Figure 2: Values of $x_{2}(x)$ and its $x_{2}^{*}(x)$ variational iteration.

where $v_{1}, v_{2}$ represent the differential variables and $v_{3}$ represents the algebraic variables. After three times of differentiation of (4.11) we have the following ODE system:

$$
\begin{gathered}
v_{1}^{\prime}=-v_{1}-v_{2}-x v_{3}+2 x, \\
v_{2}^{\prime}=-e^{x} v_{1}-(x+1) v_{2}+x^{2}+x+2, \\
v_{3}^{\prime}=0 .
\end{gathered}
$$

Differential-algebraic equation (DAE) is a Hessenberg index-3 form. 
Table 3: Numerical solution of $x_{3}(x)$.

\begin{tabular}{lccc}
\hline$x$ & $x_{3}(x)$ & $x_{3}^{*}(x)$ & $\left|x_{3}(x)-x_{3}^{*}(x)\right|$ \\
\hline 0.0 & 1 & 1 & 0 \\
0.1 & 1.195688010 & 1.195688010 & 0 \\
0.2 & 1.385683310 & 1.385683309 & $0.1 \times 10^{-8}$ \\
0.3 & 1.574816450 & 1.574816451 & $0.1 \times 10^{-8}$ \\
0.4 & 1.768554577 & 1.768554576 & $0.1 \times 10^{-8}$ \\
0.5 & 1.97381906 & 1.973081903 & $0.3 \times 10^{-8}$ \\
0.6 & 2.195390080 & 2.195390061 & $0.19 \times 10^{-7}$ \\
0.7 & 2.443379602 & 2.443379511 & $0.91 \times 10^{-7}$ \\
0.8 & 2.725973670 & 2.725973317 & $0.353 \times 10^{-6}$ \\
0.9 & 3.053245911 & 3.053244751 & $0.1160 \times 10^{-5}$ \\
1.0 & 3.436563656 & 3.436560295 & $0.3361 \times 10^{-5}$ \\
\hline
\end{tabular}

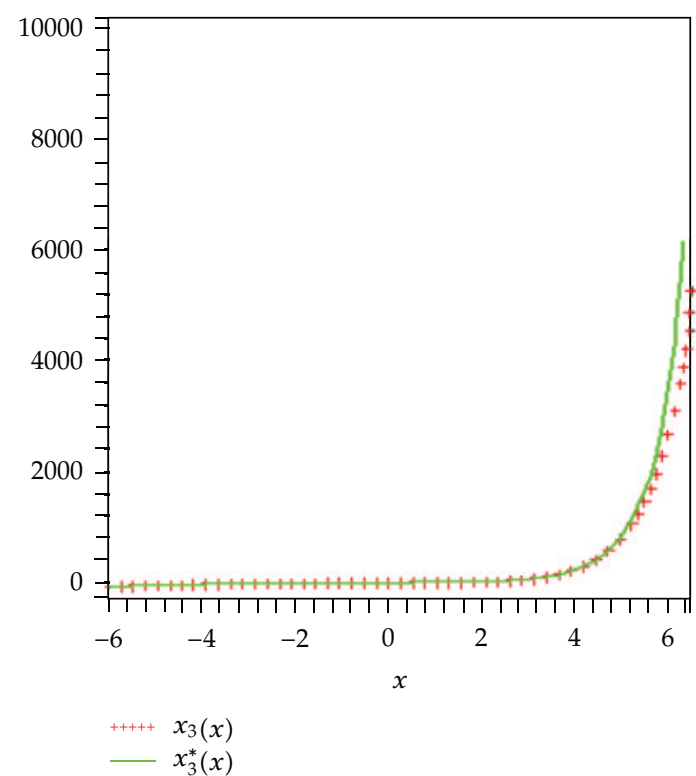

Figure 3: Values of $x_{3}(x)$ and its $x_{3}^{*}(x)$ variational iteration.

To solve system (4.14), we can construct the following correction functionals:

$$
\begin{gathered}
v_{1}^{(n+1)}(x)=v_{1}^{(n)}(x)+\int_{0}^{x} \lambda_{1}(t)\left(v_{1}^{\prime(n)}(t)+\widetilde{v}_{1}^{(n)}(t)+\widetilde{v}_{2}^{(n)}(t)+t \widetilde{v}_{3}^{()}(t)-2 t\right) d t \\
v_{2}^{(n+1)}(x)=v_{2}^{(n)}(x)+\int_{0}^{x} \lambda_{2}(t)\left(v_{2}^{\prime(n)}(t)+e^{t} v_{1}^{(n)}(t)+(t+1) \widetilde{v}_{2}^{(n)}(t)-t^{2}-t-2\right) d t \\
v_{3}^{(n+1)}(x)=v_{3}^{(n)}(x)+\int_{0}^{x} \lambda_{3}(t) v_{2}^{(n)}(t) d t
\end{gathered}
$$


Table 4: Numerical solution of $v_{1}(x)$.

\begin{tabular}{lccc}
\hline$x$ & $v_{1}(x)$ & $v_{1}^{*}(x)$ & $\left|v_{1}-v_{1}^{*}(x)\right|$ \\
\hline 0.0 & 1 & 1 & 0 \\
0.1 & 0.9048374180 & 0.9048374181 & $0.1 \times 10^{-9}$ \\
0.2 & 0.8187307531 & 0.8187307532 & $0.1 \times 10^{-9}$ \\
0.3 & 0.7408182207 & 0.7408182206 & $0.1 \times 10^{-9}$ \\
0.4 & 0.6703200460 & 0.6703200461 & $0.1 \times 10^{-9}$ \\
0.5 & 0.6065306597 & 0.6065306595 & $0.2 \times 10^{-9}$ \\
0.6 & 0.5488116361 & 0.5488116345 & $0.16 \times 10^{-8}$ \\
0.7 & 0.4965853038 & 0.4965852966 & $0.72 \times 10^{-8}$ \\
0.8 & 0.4993289641 & 0.4993289365 & $0.276 \times 10^{-7}$ \\
0.9 & 0.4065696597 & 0.4065695710 & $0.887 \times 10^{-7}$ \\
1.0 & 0.3678794412 & 0.3678791888 & $0.2524 \times 10^{-6}$ \\
\hline
\end{tabular}

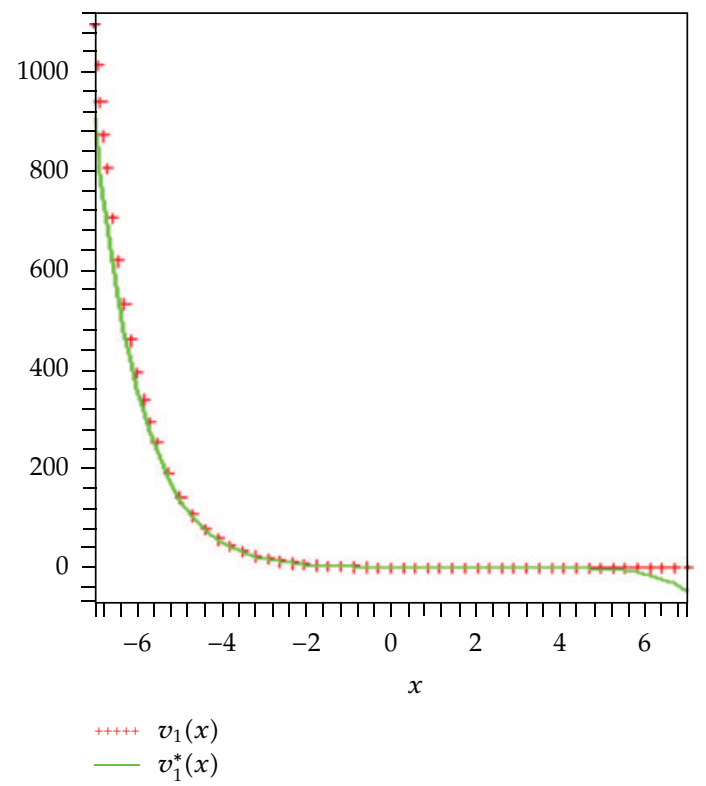

Figure 4: Values of $v_{1}(x)$ and its $v_{1}^{*}(x)$ variational iteration.

By using the basic definition of the variational iteration method can obtain that

$$
\begin{gathered}
v_{1}^{*}=1-x+\frac{1}{2} x^{2}-\frac{1}{6} x^{3}+\frac{1}{24} x^{4}-\frac{1}{120} x^{5}+\frac{1}{720} x^{6}-\frac{1}{5040} x^{7}+\frac{1}{40320} x^{8}-\frac{1}{362880} x^{9}+\cdots, \\
v_{2}^{*}=x, \\
v_{3}^{*}=1 .
\end{gathered}
$$




\section{Conclusion}

The method has been proposed for solving differential-algebraic equations with Hessenberg index-3. Results show the advantages of the method. Tables 1-4 and Figures 1-4 show that the numerical solution approximates the exact solution very well in accordance with the above method.

\section{References}

[1] K. E. Brenan, S. L. Campbell, and L. R. Petzold, Numerical Solution of Initial Value Problems in Differential-Algebraic Equations, Elsevier, New York, NY, USA, 1989.

[2] U.M. Ascher, "On symmetric schemes and differential-algebraic equations," SIAM Journal on Scientific Computing, vol. 10, no. 5, pp. 937-949, 1989.

[3] C. W. Gear and L. R. Petzold, "ODE methods for the solution of differential/algebraic systems," SIAM Journal on Numerical Analysis, vol. 21, no. 4, pp. 716-728, 1984.

[4] E. Çelik, E. Karaduman, and M. Bayram, "Numerical method to solve chemical differential-algebraic equations," International Journal of Quantum Chemistry, vol. 89, pp. 447-451, 2002.

[5] E. Çelik and M. Bayram, "On the numerical solution of differential-algebraic equations by Padé series," Applied Mathematics and Computation, vol. 137, no. 1, pp. 151-160, 2003.

[6] M. Bayram and E. Çelik, "Chebysev approximation for numerical solution of differential-algebraic equations(DAEs)," International Journal of Applied Mathematics \& Statistics, pp. 29-39, 2004.

[7] Z. Z. Ganji, D. D. Ganji, A. D. Ganji, and M. Rostamian, "Analytical solution of time-fractional NavierStokes equation in polar coordinate by homotopy perturbation method," Numerical Methods for Partial Differential Equations, vol. 26, no. 1, pp. 117-124, 2010.

[8] M. Shateri and D. D. Ganji, "Solitary wave solutions for a time-fraction generalized Hirota-Satsuma coupled KdV equation by a new analytical technique," International Journal of Differential Equations, vol. 2010, Article ID 954674, 10 pages, 2010.

[9] S. R. Seyed Alizadeh, G. G. Domairry, and S. Karimpour, "An approximation of the analytical solution of the linear and nonlinear integro-differential equations by homotopy perturbation method," Acta Applicandae Mathematicae, vol. 104, no. 3, pp. 355-366, 2008.

[10] A. R. Sohouli, M. Famouri, A. Kimiaeifar, and G. Domairry, "Application of homotopy analysis method for natural convection of Darcian fluid about a vertical full cone embedded in porous media prescribed surface heat flux," Communications in Nonlinear Science and Numerical Simulation, vol. 15, no. 7, pp. 1691-1699, 2010.

[11] J. H. He, "Variational iteration method for autonomous ordinary differential systems," Applied Mathematics and Computation, vol. 114, no. 2-3, pp. 115-123, 2000.

[12] J. Biazar and H. Ghazvini, "He's variational iteration method for solving linear and non-linear systems of ordinary differential equations," Applied Mathematics and Computation, vol. 191, no. 1, pp. 287-297, 2007.

[13] A. M. Wazwaz, "The variational iteration method for analytic treatment of linear and nonlinear ODEs," Applied Mathematics and Computation, vol. 212, no. 1, pp. 120-134, 2009.

[14] D. D. Ganji, E. M. M. Sadeghi, and M. Safari, "Application of He's variational iteration method and adomian's decom- position method method to Prochhammer Chree equation," International Journal of Modern Physics B, vol. 23, no. 3, pp. 435-446, 2009.

[15] M. Safari, D. D. Ganji, and M. Moslemi, "Application of He's variational iteration method and Adomian's decomposition method to the fractional KdV-Burgers-Kuramoto equation," Computers $\mathcal{E}$ Mathematics with Applications, vol. 58, no. 11-12, pp. 2091-2097, 2009.

[16] D. D. Ganji, M. Safari, and R. Ghayor, "Application of He's variational iteration method and Adomian's decomposition method to Sawada-Kotera-Ito seventh-order equation," Numerical Methods for Partial Differential Equations, vol. 27, no. 4, pp. 887-897, 2011. 


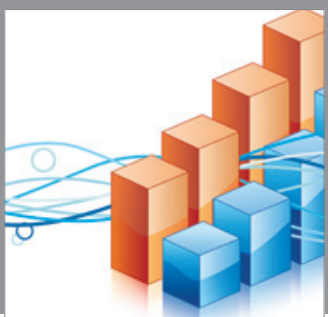

Advances in

Operations Research

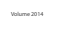

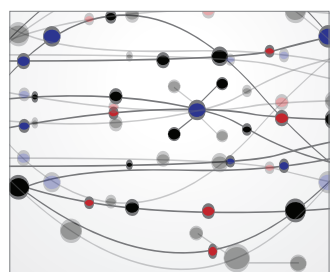

\section{The Scientific} World Journal
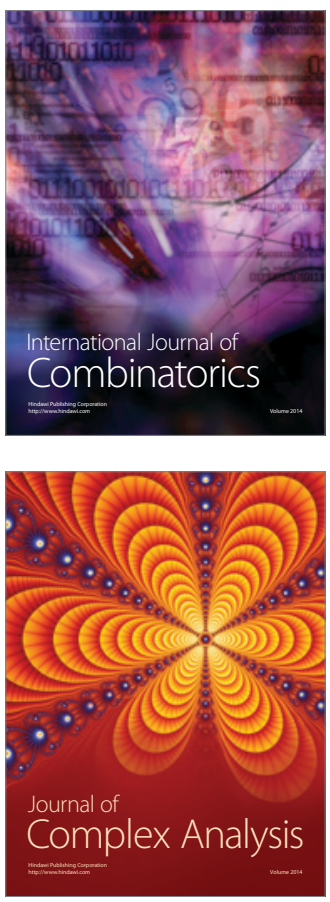

International Journal of

Mathematics and

Mathematical

Sciences
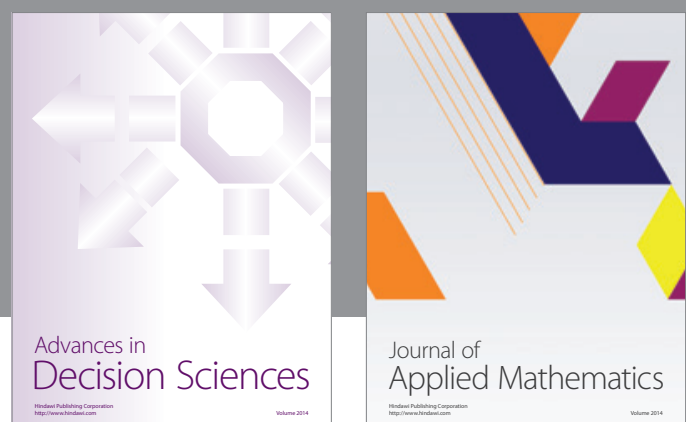

Journal of

Applied Mathematics
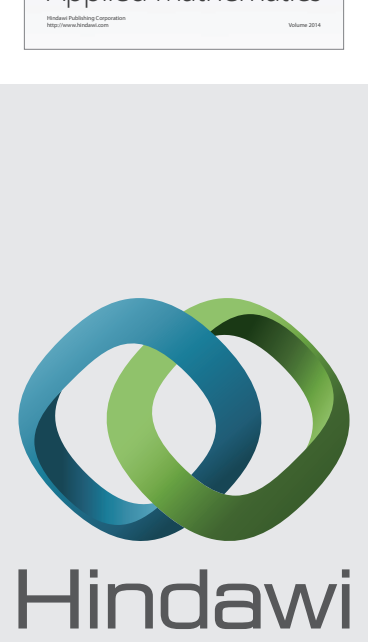

Submit your manuscripts at http://www.hindawi.com
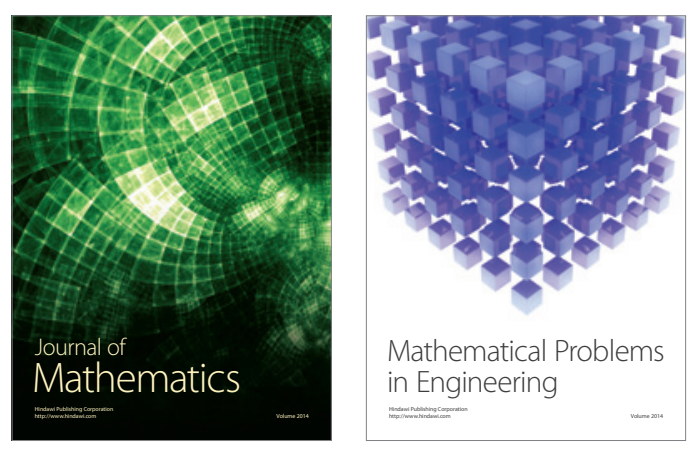

Mathematical Problems in Engineering
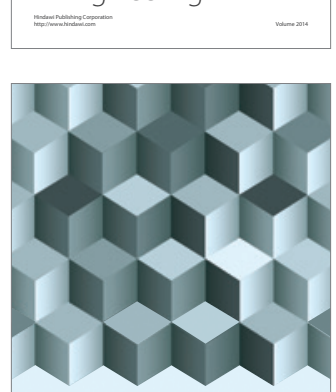

Journal of

Function Spaces
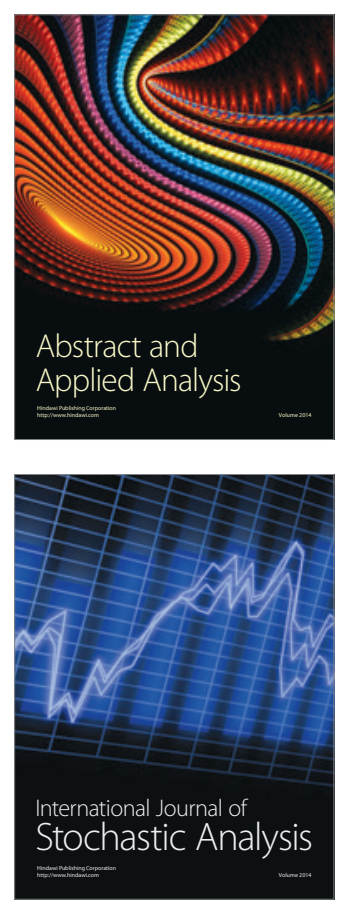

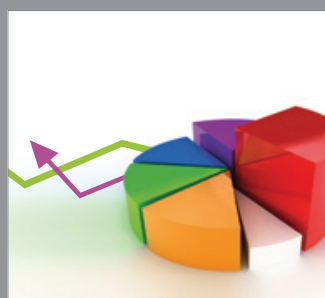

ournal of

Probability and Statistics

Promensencen
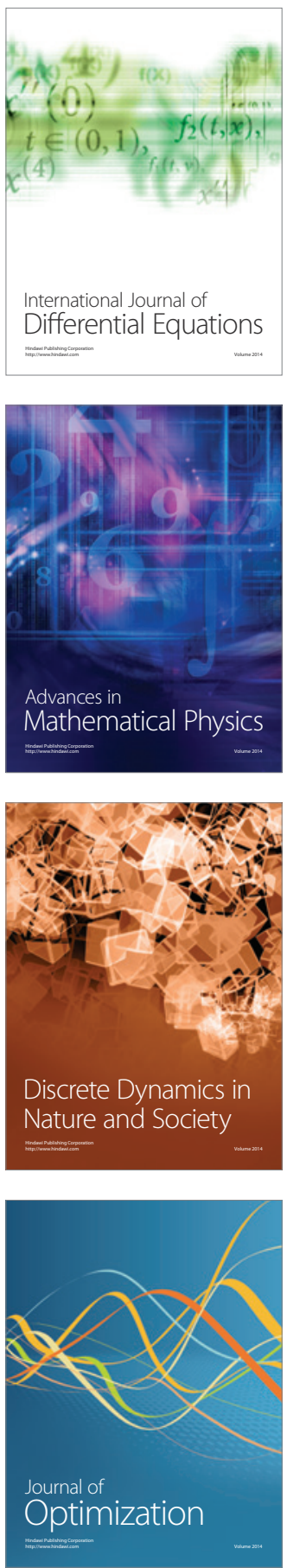